\title{
Наносетчатые пленки из углеродных нанотрубок с X-соединениями для электронных и фотовольтаических приложений
}

\author{
(C) О.Е. Глухова, М.М. Слепченков , К.Р. Асанов \\ Саратовский национальный исследовательский государственный университет им. Н.Г. Чернышевского, \\ 410012 Саратов, Россия \\ ๑ E-mail: slepchenkovm@mail.ru
}

Поступила в Редакцию 17 февраля 2020 г.

В окончательной редакции 3 августа 2020 г.

Принята к публикации 10 августа 2020 г.

\begin{abstract}
Исследованы атомная структура, электронные и оптоэлектронные свойства наносетчатых пленок из углеродных нанотрубок с бесшовными крестообразными Х-соединениями. Установлено, что топология расположения негексагональных элементов в области контакта нанотрубок определяет энергетическую стабильность атомной структуры. Выявлено, что размеры поры пленки определяют тип проводимости. При наименьших размерах поры пленка характеризуется металлическим типом проводимости, с увеличением размера поры щель в зонной структуре увеличивается и пленка становится полупроводниковой. Пленки с минимальным размером щели имеют хорошие фотовольтаические характеристики. Фототок для рассмотренных моделей пленки может достигать $2.4 \mathrm{MA} \cdot \mathrm{cm}^{-2}$ в условиях атмосферы и $3.25 \mathrm{MA} \cdot \mathrm{cm}^{-2}$ вне атмосферы. Наличие щели в зонной структуре делает наносетчатые пленки перспективными для нано- и оптоэлектроники.
\end{abstract}

Ключевые слова: наносетки, углеродные нанотрубки, спектр поглощения, электропроводность, фототок.

DOI: $10.21883 /$ FTP.2020.12.50237.9372

\section{1. Введение}

Наносетчатые пленки из углеродных нанотрубок (НСПУНТ) с бесшовными крестообразными Х-соединениями, которые часто называют суперквадратными сетками, являются до сих пор малоизученным объектом в связи с технологическими сложностями их получения. Однако в последнее десятилетие в этом направлении наблюдается большой прогресс. Углеродные нанотрубки (УНТ) укладываются в определенном порядке и в виде определенного рисунка $[1,2]$. Одним из ярких достижений в этой области стала методика получения углеродных пленок и наноструктур с контролируемыми размером, формой и позиционированием УНТ друг относительно друга [3], а также лазерный способ наносварки сетей из УНТ с бесшовным соединением [4]. В этих работах показана возможность не только аккуратной укладки УНТ на подложке, но и формирования бесшовных $\mathrm{X}$-соединений с заданными размерами сот наносетки. В другой работе показан очень эффективный способ наносварки УНТ с образованием пористого наноматериала с бесшовными V-, Y-, звездочными и крестообразными $\mathrm{X}$-соединениями [5]. Методом наносварки формируются также прочные гибридные наноматериалы графен-УНТ с бесшовными контактами нанотрубок и графена, а также пористые углеродные структуры [6]. Необходимость бесшовных соединений диктуется фактом снижения проводимости и прочности при слабых контактах между трубками в составе наносеток и трехмерных (3D) наноматериалов [7]. Необходимость получения пленок типа НСПУНТ диктуется задачами современной электроники. Подобные пленки востребованы прежде всего в гибкой и растяжимой электронике, одной из актуальных задач которой являются обеспечение электронных наноустройств эластичными дисплеями [8-10] и биомедицинских устройств сенсорами, выдерживающими многократные деформации изгиба, растяжения/сжатия $[11,12]$. Пленки типа НСПУНТ отличаются необыкновенной механической прочностью на фоне высокой эластичности, а также высокой стабильностью и устойчивостью к внешним воздействиям [13]. Наноматериалы с бесшовными соединениями УНТ-УНТ выдерживают нагрузки на сжатие до $72 \mathrm{MПа,} \mathrm{на} \mathrm{изгиб} \mathrm{до} 33$ МПа и выдерживают 100000 циклов сжатия, восстанавливая исходную структуру [5]. Все это предопределило большие перспективы применения их в качестве мембран [14]. Мембраны на основе НСПУНТ имеют превосходные характеристики опреснения воды, выступая в качестве нового типа нанопористых мембран, эффективно задерживающих $\mathrm{NaCl}$ из воды. Гибкость подобной пленки позволяет ей выдержать большие локальные напряжения, вызываемые значительным давлением фильтруемой воды. При этом эффективность работы мембраны в процессе фильтрации определяется размером ее пор. Более широкие перспективы применения НСПУНТ требуют глобальных экспериментальных и теоретических исследований. Отличие этих двумерных (2D) наноматериалов от обычных пленок с хаотичным расположением УНТ заключается в возможности появления анизотропии проводимости, контролируемом характере проводимости, а также перспективности применения в фотовольтаике. Действительно, при использовании НСПУНТ из-за бесшовности Х-соединений отсутствуют препятствия для квантового транспорта электронов в области контакта 
трубок. В случае обычных пленок из смеси беспорядочно расположенных УНТ наблюдаются межтрубные контакты, электрическое сопротивление которых составляет сотни Ом и даже единицы МОм [15]. К тому же НСПУНТ предусматривают возможность формирования бесшовных Х-соединений из трубок разной хиральности, что предопределяет различные свойства в разных направлениях, в том числе разную удельную электропроводность и динамическую проводимость как чувствительность пленок к электромагнитным волнам разных частот и разной поляризации. Контроль размеров сот, образованных соединениями УНТ-УНТ в НСПУНТ, позволяет управлять механическими свойствами $[13,15]$. При этом не исключена возможность контроля транспортных, оптических и электродинамических свойств. Эти вопросы требуют отдельных исследований, поскольку ответы на них могут открыть новые физические явления в НСПУНТ и их применения.

В данной работе in silico исследуется размерный эффект НСПУНТ на основе одностенных УНТ $(16,0)$, заключающийся во влиянии размера соты (окна, образованного контактами трубок) на электронную структуру, проводимость, спектр и величину фототока. Трубки $(16,0)$ были выбраны в связи с тем, что: именно эти трубки относятся к наиболее часто синтезируемым [16]; благодаря малому периоду трансляции атомной сетки этих трубок (в отличие от хиральных) можно проводить исследования наносетчатых пленок, применяя высокоточные квантовые методы. Также отметим, что именно одностенные УНТ (ОУНТ) с полупроводниковым типом проводимости составляют подавляющее большинство среди синтезируемых трубок.

\section{2. Атомная и электронная структура}

Для построения атомистических моделей суперъячеек НСПУНТ был применен оригинальный метод автоматического генерирования моделей разветвленных структур произвольной формы с бесшовными контактами, в том числе из углеродных наноструктур, разработанный ранее при участии авторов данной работы [17]. Этот метод реализован в оригинальной программе SeamMaker [17], также разработанной при участии авторов. Кратко, указанный метод включает геометрический и динамический этапы. Геометрический этап заключается в построении триангулированной наносетки, предназначенной служить первоначальным каркасом для соединения двух рассматриваемых структур. В общем случае наносетка-каркас представляет собой усеченный конус, основания которого принадлежат соединяемым нанообъектам. Динамический этап использует молекулярную динамику для формирования контакта на основании информации, получаемой на геометрическом этапе. При этом используется силовое поле, наиболее подходящее для данных исследуемых наноструктур, в нашем случае - AIREBO [18]. На этом этапе может применяться термостат при необходимости обеспечения определенной заданной температуры, а также баростат. Возможна реализация динамического этапа без термостата и баростата. В данной работе применялись нормальные условия, т.е. нормальное давление 105 Па и температура $300 \mathrm{~K}$. Шаг интегрирования по времени брался равным 0.1 фс. В рамках указанного нового метода для ускорения процесса формирования области контакта между двумя наноструктурами применяется модифицированное силовое поле на базе AIREBO. Суть модификации заключается во введении дополнительного члена в уравнение движения, которое принимает следующий вид:

$$
\frac{d \mathbf{r}_{i}(t)}{d t^{2}}=-\nabla_{i} U\left(\mathbf{r}_{1}, \ldots, \mathbf{r}_{N}\right)+\mathbf{F}_{\mathrm{ext}}
$$

В уравнении (1) дополнительным членом является сила $\mathbf{F}_{\text {ext }}$, притягивающая внешний атом к поверхности наносетки-каркаса, построенной на геометрическом этапе. В качестве потенциала $U$, как упоминалось выше, выбран потенциал AIREBO. Выражение для силы $\mathbf{F}_{\mathrm{ext}}$ имеет следующий вид:

$$
\mathbf{F}_{\mathrm{ext}}=k d^{2} \mathbf{e},
$$

где $k$ - коэффициент, обеспечивающий ту величину силы, которая приводит к быстрому приближению внешнего дополнительного атома к поверхности каркаса (дополнительные атомы необходимы для построения соединения между рассматриваемыми наноструктурами). В результате серии численных экспериментов величина $k$ была определена и составила $100 \mathrm{HH} / \mathrm{A}^{2}$ [17]. Таким образом, к каждому внешнему атому приложена своя сила $\mathbf{F}_{\text {ext }}$, под действием которой атомы устремляются к каркасу и по мере приближения к нему уже начинают испытывать действие силового поля AIREBO.

C помощью программы SeamMaker было построено 5 разных моделей крестообразных Х-соединений из одностенных УНТ $(16,0)$. Все 5 моделей различаются набором негексагональных элементов и топологией их размещения в области Х-образного контакта. Все модели выгодны энергетически и являются стабильными структурами. Размеры суперъячейки во всех случаях составляют $2.805 \times 2.982$ нм. Это наименьшие размеры, при которых суперъячейка для случая ОУНТ $(16,0)$ является равновесной структурой и энергетически стабильной. На рис. 1 представлены три модели $(1,2,3)$, характеризующиеся наименьшей потенциальной энергией $E_{\text {pot }}$ (в расчете на атом), но имеющие разный набор пентагонов/гептагонов/октагонов. Также они различаются характером их распределения в области Х-образного контакта и количеством атомов в суперьячейке: модель 1 имеет 616 атомов, модель $2-614$ атомов, модель $3-$ 615 атомов. Кривые на рис. 1 показывают убывание потенциальной энергии равновесной структуры в ходе молекулярно-динамического моделирования Х-образного контакта нанотрубок в программе SeamMaker. Как 


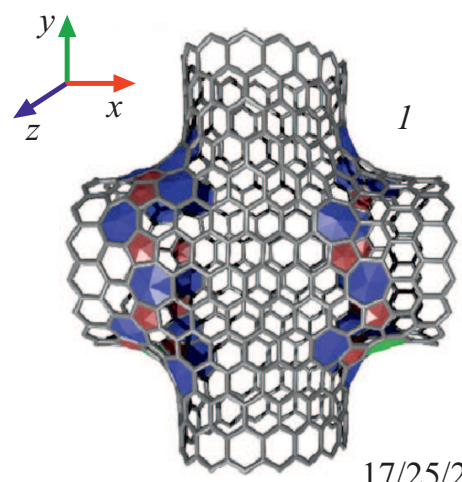

$17 / 25 / 2$
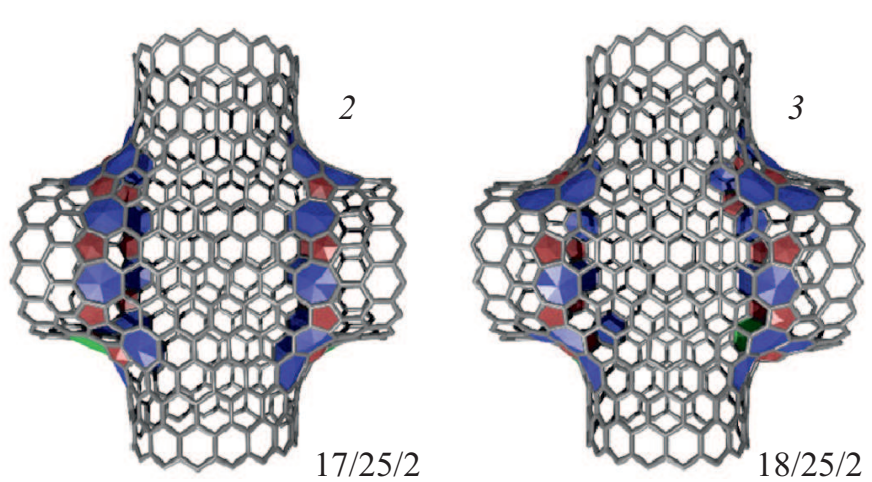

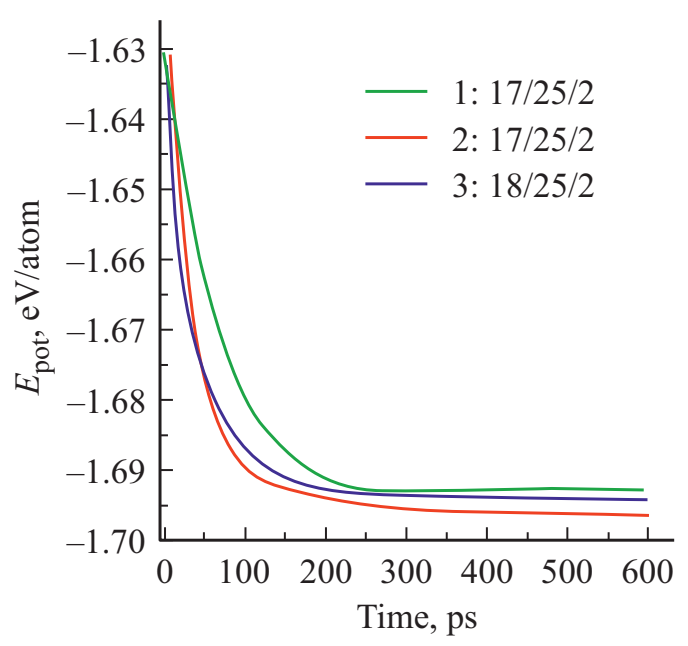

Рис. 1. Три атомистические модели суперьячеек НСПУНТ на основе ОУНТ $(16,0)$ с различным количеством и топологией расположения негексагональных элементов пентагон/гептагон/октагон.

видно, наименьшей энергией характеризуется модель 2. При этом стабильные энергетически структуры получаются уже в первые 200-300 пс моделирования. Далее атомная структура уточняется, но уже в небольших пределах.

Далее была проведена реоптимизация атомной структуры всех пяти моделей с помощью квантово-механического метода функционала плотности в приближении сильной связи SCC DFTB2 [19]. Этот метод корректно (с физической точки зрения) воспроизводит электронные свойства наноструктур, суперъячейки которых содержат несколько сотен и даже тысячи атомов [20,21]. При этом проводилась двойная реоптимизация, подразумевающая одновременную оптимизацию атомной структуры суперъячейки и периодического ящика. Таким образом, параметрами оптимизации выступали не только координаты атомов, но и векторы трансляций $L_{x}, L_{y}$ (метрические параметры периодического ящика). Величины энергии на атом составляют $-47.185,-47.238$ и -47.226 эВ соответственно. Можно видеть, что модели 1 и 2, имеющие одинаковой набор негексагональных элементов, различаются по энергии, рассчитанной в рамках квантового метода, как и в случае потенциальной энергии, рассчитанной молекулярно-динамическим методом.
Это говорит об определяющей роли топологии атомной сетки. По итогам проведенных построений был сделан выбор в пользу самой выгодной с энергетической точки зрения модели 2. Именно НСПУНТ, построенные на базе этой суперъячейки, далее рассматриваются детально, в частности исследуются их электропроводность и оптоэлектронные свойства.

На базе выбранной модели НСПУНТ построены шесть моделей с топологически одинаковыми Х-соединениями нанотрубок, различающимися только размерами поры. Размеры поры определяются величинами векторов трансляций в направлениях $X$ и $Y$, как показано на рис. 2, $a$, где представлена модель НСПУНТ с наименьшей порой, имеющей практически квадратную форму и размеры $a \times b=1.55 \times 1.86$ нм. Эта модель отвечает наименьшей суперъячейке, являющейся энергетически стабильной, как отмечалось выше. На базе именно этой модели строились все остальные путем постепенного наращивания длины нанотрубок кольцами из гексагонов в обоих направлениях $X$ и $Y$. Они будут обозначаться как НСПУНТ- $N$ (где $N$ - количество колец гексагонов). Число $N=0$ отвечает начальной конфигурации с наименьшей порой (рис. 2,a). Такой способ наращивания позволил обеспечить квадратную форму поры. 

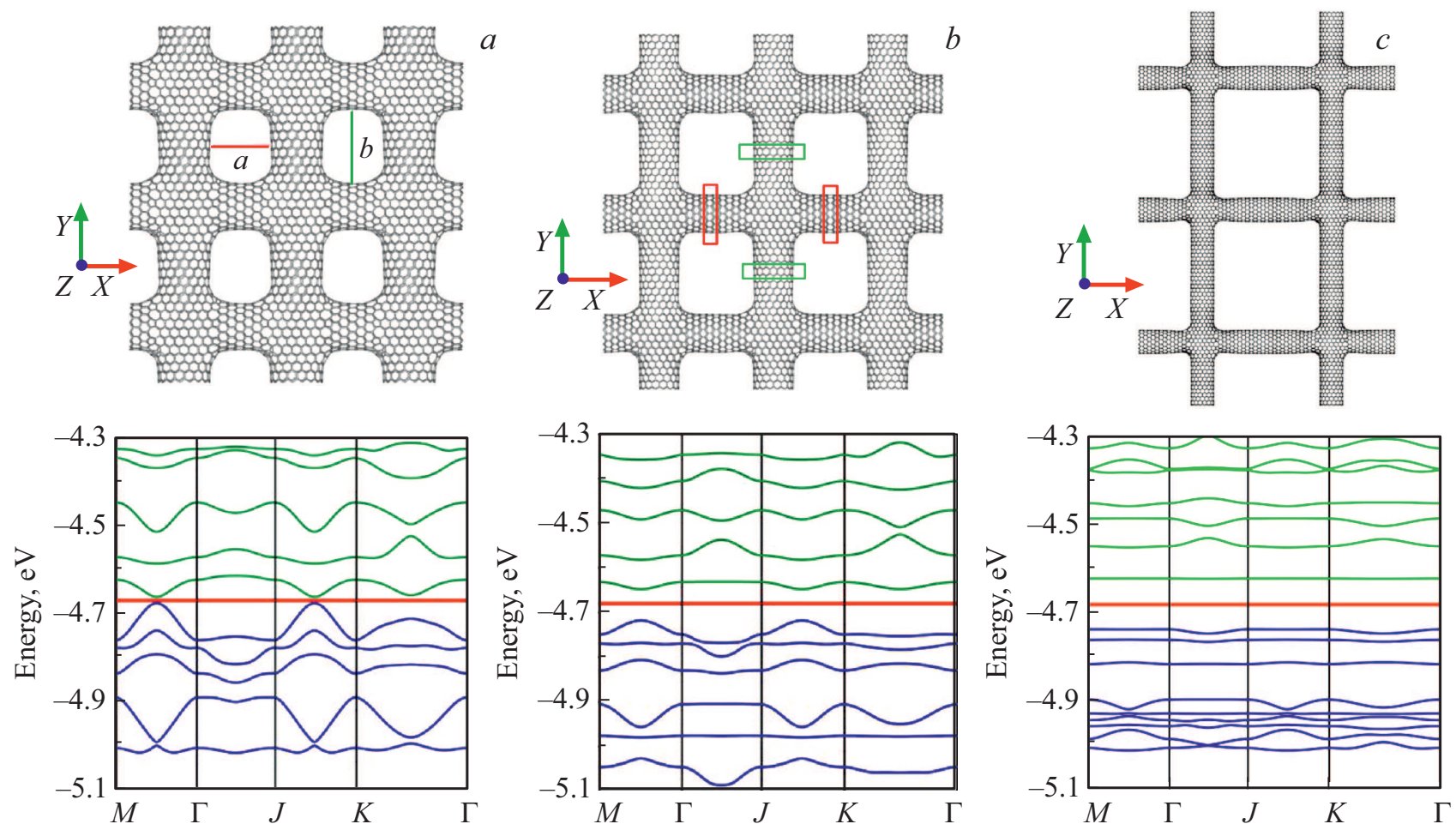

Рис. 2. Атомная и зонная структура НСПУНТ- $N$ с минимальным $(a, N=0)$, увеличенным $(b, N=1)$ и максимальным размером поры $(c, N=5)$.

Одинаковость формы поры необходима, для того чтобы исключить влияние фактора топологии формы поры на исследуемые свойства. На рис. 2, $b$ показана атомная структура модели НСПУНТ- $N$ для случая наращивания тубуса нанотрубки на одно кольцо из гексагонов $(N=1)$. Эти кольца из гексагонов выделены для наглядности прямоугольниками. Каждый случай послойного наращивания длины нанотрубок и соответственно увеличения размеров поры $a \times b$ сопровождался реоптимизацией атомной структуры, а также расчетами электронной зонной структуры пленки. На рис. 2, с показана атомная структура НСПУНТ-5, которая имеет максимальные размеры поры среди всех рассматриваемых в данной работе структур. В табл. 1 представлены метрические и энергетические характеристики шести моделей НСПНУТ, построенных на базе выбранной структуры Х-образного соединения (структура 2 на рис. 1). Все модели имеют отрицательную энтальпию формирования, равную $-(0.13 \pm 0.04)$ ккал/моль - атом. Размер поры, как видно из табл. 1, постепенно увеличивается от 1.5 до 6 нм. Увеличение размеров поры сопровождается изменением зонной структуры и электронных свойств.

Из табл. 1 видно, как с увеличением размеров поры уровень Ферми $E_{\mathrm{F}}$ медленно смещается от -4.67 до -4.69 эВ. Это изменение незначительно, однако с позиции зонной структуры происходят гораздо бо́льшие изменения. Энергетическая щель $E_{\text {gap }}$ между валентной зоной и зоной проводимости резко увеличивается от практически нулевого значения до 0.117 эВ. Действи-
Таблица 1. Метрические и энергетические характеристики шести моделей НСПНУТ, построенных на базе выбранной структуры Х-образного соединения

\begin{tabular}{|c|c|c|c|c|c|c|}
\hline$N$ & $L_{x}, \mathrm{HM}$ & $L_{y}, \mathrm{HM}$ & $a, \mathrm{HM}$ & $b, \mathrm{HM}$ & $E_{\mathrm{F}}$, эB & $E_{\text {gap }}, \ni \mathrm{B}$ \\
\hline 0 & 2.81 & 2.98 & 1.55 & 1.86 & -4.674 & 0.001 \\
\hline 1 & 3.66 & 3.83 & 2.41 & 2.58 & -4.675 & 0.071 \\
\hline 2 & 4.51 & 4.68 & 3.26 & 3.43 & -4.680 & 0.085 \\
\hline 3 & 5.36 & 5.55 & 4.11 & 4.3 & -4.682 & 0.105 \\
\hline 4 & 6.21 & 6.39 & 4.96 & 5.14 & -4.687 & 0.114 \\
\hline 5 & 7.07 & 7.24 & 5.82 & 5.95 & -4.692 & 0.117 \\
\hline
\end{tabular}

тельно, отсутствие заметной энергетической щели в случае $N=0$ наблюдается в зонной структуре, представленной на рис. 2, $a$. Зонная структура представлена с учетом симметрии 2D зоны Бриллюэна и включает точки симметрии $M\left(\pi / L_{x}, 0\right), \Gamma(0,0), J\left(0, \pi / L_{y}\right)$ и $K\left(\pi / L_{x}, \pi / L_{y}\right)$. Из рис. 2, a видно, как потолок валентной зоны и дно зоны проводимости смыкаются на двух участках между точками $M$ и $\Gamma, J$ и $K$. Однако уже для модели НСПУНТ-1 $(N=1)$ наблюдается резкое изменение картины подзон (рис. $2, b$ ). Возникает щель. Этот факт не является удивительным, поскольку трубки $(16,0)$ являются полупроводниковыми и неизбежно должны проявить свои свойства в наносетчатой пленке. Как раз удивительным является тот факт, что для случая $N=0$, когда размеры поры минимальны, щель 

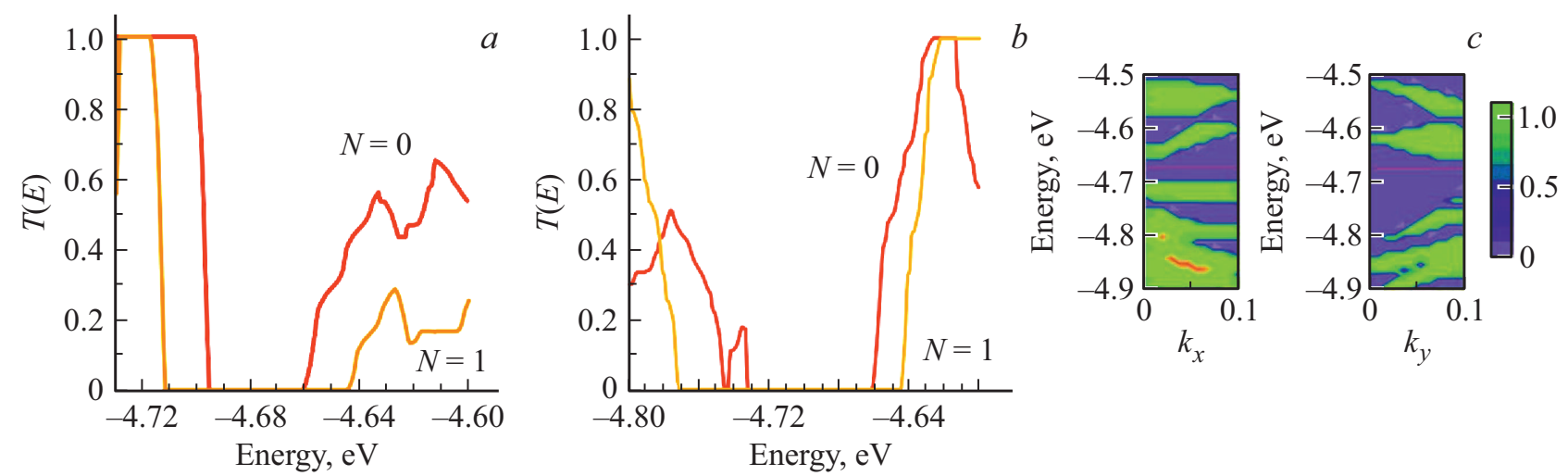

Рис. 3. Интегральная функция пропускания для НСПУНТ-0 и НСПУНТ-1 при токопереносе в направлении оси $X$ (a) и в направлении оси $Y(b)$, карты функции проводимости $T(E, k)$ для НСПУНТ-0 $(c)$.

отсутствует, и пленка является высоко проводящей. Это можно объяснить большим влиянием на электронное строение негексагональных элементов в области контакта нанотрубок. По мере наращивания длины нанотрубок и увеличения размеров поры влияние области контакта уменьшается и пленка проявляет полупроводниковые свойства. При максимальном из рассмотренных размеров пор (рис. 2,c) дно зоны проводимости становится ровным для всех участков зоны Бриллюэна, и щель достигает максимального значения.

\section{3. Электропроводность}

Для исследования закономерностей электропроводности НСПУНТ применяется аппарат неравновесных функций Грина-Келдыша и формализм ЛандауэраБуттикера [22], который позволяет рассчитать электропроводность на основе функции пропускания электронов $T(E)$ :

$$
G=\frac{2 e^{2}}{h} \int_{-\infty}^{\infty} T(E) F_{T}\left(E-E_{\mathrm{F}}\right) d E
$$

где $e-$ заряд электрона, $h$ - постоянная Планка. Величина $e^{2} / h$ - квант проводимости, величина для единственного канала проводимости. Это значение удваивается для учета спина электронов. Функция теплового уширения $F_{T}(E)$ рассчитывается по формуле

$$
F_{T}(E)=\frac{1}{4 k_{\mathrm{B}} T} \operatorname{sech}\left(\frac{E}{2 k_{\mathrm{B}} T}\right),
$$

где $k_{\mathrm{B}}-$ постоянная Больцмана, $T-$ температура. Функция пропускания электронов, как известно, характеризует квантово-механическую прозрачность проводящего канала (участка структуры, заключенного между двумя контактами) в зависимости от энергии движущегося по нему электрона. Используемый формализм предусматривает описание закономерностей когерентного транспорта электронов, когда при движении электрона через канал учитываются только упругие столкновения с атомами кристаллической решетки. Если речь идет о 2D наноматериале, как в рассматриваемом случае, функция пропускания определяется не одной энергией, а сразу двумя параметрами - энергией электрона и его состоянием, т.е. волновым числом $k$ зоны Бриллюэна. Процедура расчета функции пропускания $T(E, k)$ является весьма ресурсоемкой, особенно в случае многоатомных суперъячеек. В связи с этим ранее авторами данной работы была разработана методика ускорения расчета $T(E)$, которая подробно представлена в работе [23]. Новизна и перспективность методики заключается в возможности восстановления истинного профиля поверхности 2D функции пропускания электронов $T(E)$, рассчитываемой для разных значений энергии $E$ и различных состояний $k$, по результатам расчетов $T(E, k) \mathrm{c}$ малым разбиением первой зоны Бриллюэна. По данным профиля поверхности $T(E, k)$ уже рассчитывается интегральная функция пропускания $T(E)$, фигурирующая в формуле (1). Интегральная функция пропускания $T(E)$ рассчитывается усреднением по всем $k$. Разработанная методика была апробирована на расчетах $T(E)$ для хорошо изученных 2D материалов типа графена и графана. B данной работе она применена для 2D наносетчатых структур из Х-соединений ОУНТ.

На рис. 3 представлены результаты расчетов интегральной функции пропускания для направлений транспорта электронов вдоль осей $X$ и $Y$ : на рис. $3, a-$ $T(E)$ для НСПУНТ-0 и НСПУНТ-1 для направления $X$, на рис. $3, b$ - для направления $Y$. Единицы измерения функции пропускания - кванты проводимости $e^{2} / h$. В обоих случаях канал проводимости, когда $T(E)=1$, не достигается на уровне Ферми, $\sim(-4.67)$ эВ, где, наоборот, отмечается область нулевых значений $T(E)$. Область нулевых значений $T(E)$ для модели НСПУНТ-0 наблюдается только в узком интервале энергий. Для модели НСПУНТ-1 этот интервал расширяется, что объясняется увеличением ширины запрешенной зоны 
по мере увеличения размеров поры (табл. 1). Помимо разницы в спектрах функции $T(E)$, для моделей НСПУНТ-0 и НСПУНТ-1 наблюдается заметная разница в закономерностях квантового транспорта электронов для одной и той же модели в зависимости от направления $X$ или $Y$. Для направления $X$ (рис. $3, a$ ) характерно меньшее значение нулевого участка функции $T(E)$, чем для направления $Y$, для обеих моделей, НСПУНТ-0 и НСПУНТ-1. Объяснение такой разницы можно увидеть на карте $T(E, k)$ для НСПУНТ-0, представленной на pис. $3, c$. Если для направления $X$ функция $T\left(E, k_{x}\right)$ имеет значения 1 непосредственно вблизи уровня Ферми, то для направления $Y$ на карте $T\left(E, k_{y}\right)$ можно видеть обширную область минимальных значений. По картам зонной структуры, представленным на рис. $2, a, b$, можно увидеть, что в направлении $X$ (участок $\Gamma-M$ ) щель между валентной зоной и зоной проводимости заметно меньше по сравнению с направлением $Y$ (участок $\Gamma-J)$. Все эти факты однозначно предопределяют более выгодное направление транспорта электронов в направлении $X$. Рассчитанные электрические сопротивления имеют следующие значения для НСПУНТ-0: $R_{x}=56.3$ и $R_{y}=156.7$ кОм ·нм. При увеличении поры вместе с увеличением энергетической щели зонной структуры (табл. 1) возрастает и электрическое сопротивление до 800-1000кОм·нм. Если сравнивать полученные значения сопротивлений с аналогичными значениями для трубок с металлическим типом проводимости $(R=1.5 \kappa О м \cdot$ нм для трубок типа „кресло“ $)$, то в данном случае превышение очень значительно. Однако, как отмечалось выше, ОУНТ с полупроводниковым типом проводимости составляют подавляющее большинство среди синтезируемых трубок. Трубки с металлическим типом проводимости составляют $\sim(10-20) \%$. Наличие щели на уровне Ферми в профиле интегральной функции пропускания $T(E)$ указывает на перспективность применения НСПУНТ для изготовления транзисторов и многотерминальных устройств. Важным моментом является также открытая в данной работе возможность управления величиной этой щели с помощью регулирования размеров пор.

\section{4. Фотовольтаические свойства}

Для исследования фотовольтаических свойств НСПУНТ был рассчитан в первую очередь тензор комплексной динамической проводимости $\sigma_{\alpha \beta}(\Omega)$ в ультрафиолетовом-видимом-инфракрасном (УФ-видимом-ИК) диапазоне, на основании которого далее рассчитывается спектр поглощения и спектр фототока. Отметим, что в зарубежной литературе вместо термина „динамическая проводимость“ применяется „оптическая проводимость“ из-за исследуемого диапазона длин волн. Для расчета тензора $\sigma_{\alpha \beta}(\Omega)$ применялась известная формула Кубо-Гринвуда, определяющая проводимость как функцию энергии фотона $\Omega$ и которая может быть записана в виде [24]

$$
\begin{aligned}
\sigma_{\alpha \beta}= & \frac{2 e^{2} \hbar}{i m_{e}^{2} S_{\text {cell }}} \frac{1}{N_{\mathbf{k}}} \sum_{\mathbf{k} \in B Z}^{N_{\mathbf{k}}} \sum_{m, n} \frac{\hat{P}_{\alpha}^{n, m}(\mathbf{k}) \cdot \hat{P}_{\beta}^{n, m}(\mathbf{k})}{E_{n}(\mathbf{k})-E_{m}(\mathbf{k})+\Omega+i \eta} \\
& \times \frac{f_{\beta}\left[E_{n}(\mathbf{k})-\mu\right]-f_{\beta}\left[E_{m}(\mathbf{k})-\mu\right]}{E_{n}(\mathbf{k})-E_{m}(\mathbf{k})},
\end{aligned}
$$

где $f_{\beta}(E)=1 /(1+\exp [\beta(E-\mu)])-$ функция ФермиДирака с химическим потенциалом $\mu, \beta=1 / k_{\mathrm{B}} T ; S_{\text {cell }}-$ площадь поперечного сечения суперьячейки; $k_{\mathrm{B}}-$ постоянная Больцмана, $N_{\mathbf{k}}$ - количество k-точек зоны Бриллюэна; $\hat{P}_{\alpha}^{n m}(\mathbf{k})$ элементы матрицы операторов момента импульсов, соответствующие $\alpha$-компоненте вектора импульса; $\hat{P}_{\beta}^{n m}(\mathbf{k})$ - элементы матрицы операторов момента импульсов, соответствующие $\beta$-компоненте вектора импульса; $m_{e}$ и $e-$ масса и заряд электрона; $E_{n}(\mathbf{k})$ - энергия подзон для валентной зоны, $E_{m}(\mathbf{k})-$ энергия подзон для зоны проводимости. Спин электрона учитывается числом 2. Феноменологический параметр $\eta$ характеризует рассеяние электронов. Для расчета элементов матрицы импульсов $\hat{P}_{\alpha}^{n m}(\mathbf{k})$ использовалось известное соотношение $\hat{P}(\mathbf{k}) \rightarrow\left(m_{e} / \eta\right) \nabla_{\mathbf{k}} \hat{H}(\mathbf{k})$, где $\hat{H}(\mathbf{k})$ - гамильтониан. Гамильтониан строился в рамках метода SCC DFTB2. Расчет коэффициента поглощения $A(\omega)$ в данном диапазоне длин волн рассчитывался по формуле

$$
A(\omega)=l-|R(\omega)|^{2}-|T(\omega)|^{2},
$$

где $R(\omega)=-\sigma_{\alpha \beta} Z_{0} /\left(2+\sigma_{\alpha \beta} Z_{0}\right)$ - коэффициент отражения, $T(\omega)=2 /\left(2+\sigma_{\alpha \beta} Z_{0}\right)-$ коэффициент пропускания, $Z_{0}-$ волновое сопротивление, равное величине $120 \pi$ Ом для случая вакуума и нормального падения световых волн. Применяемые формулы для коэффициентов отражения и пропускания справедливы для тонких пленок, толщина которых много меньше длины волны падающего излучения [25]. В рассматриваемом случае толщина пленки $\sim 2 \mathrm{HM}$, что намного меньше длин волн изучаемого диапазона 100-2000 нм. Заметим, что солнечный свет не поляризован, поэтому вычисляется среднее значение $A(\omega)$ для волн $s$ - и $p$-поляризации. Спектр фототока может быть рассчитан с помощью следующей формулы [26]:

$$
I_{\max }(\omega)=\frac{e P_{\text {in }}}{\eta \omega} A(\varphi),
$$

где $P_{\text {in }}$ - мощность падающего излучения. Формула (5) позволяет получить спектр максимального фототока, так как не учитывает частичную рекомбинацию электронов и дырок. Ее можно учесть, но для этого должна рассчитываться функция пропускания $T(E)$ в рамках формализма неупругого транспорта электронов, что в данной работе не предусматривается. Таким образом, формула (5) применима для случая внутреннего квантового выхода 100\%, когда каждый поглощенный фотон порождает электрон. На практике эта величина составляет 85-90\% [26]. 



Рис. 4. Спектры поглощения $A$ НСПУНТ $(a)$ и спектры фототока $I_{\mathrm{ph}}$ в условиях атмосферы Земли АМ1.5 $(b)$, вне атмосферы AM0 $(c)$. Сверху вниз $N=0,1,2,3,4,5$. Нижняя кривая $(a)$ - расчетный спектр поглощения пленки из одинаковых трубок $(16,0)$, расположенных на расстоянии ван-дер-ваальсового взаимодействия.

Рассчитанные спектры поглощения для интервала длин волн 100-2000 нм для всех моделей НСПУНТ- $N$ представлены на рис. 4, $a$. По мере увеличения размеров поры коэффициент поглощения во всем диапазоне длин волн уменьшается. Эта тенденция особенно хорошо заметна для моделей НСПУНТ-0,1,2. Почти равномерно уменьшается величина. Выделяются случаи НСПУНТ-3,4,5. Для них характерно появление резко выделяющихся высоких пиков интенсивности в ИК области в интервалах 900-1000, 1300-1500, 1700-1800 нм, а также в видимой области 500-650нм. Объяснением такого изменения в профиле спектра поглощения для НСПУНТ-3,4,5 служит значительное изменение размеров пор для этих моделей. На рис. 2, с при размере поры $\sim 6 \times 6$ нм видно, что размеры тубуса нанотрубок значительно превышают размеры областей контакта трубок, что и приводит к постепенному превалированию поглощательных свойств самих трубок по сравнению со свойствами областей контакта. Для того чтобы в этом убедиться, мы рассчитали спектр поглощения пленки из одинаковых трубок $(16,0)$, расположенных на расстоянии ван-дер-ваальсового взаимодействия, этот спектр представлен на рис. 4, $a$ (правая шкала $A(\omega)$ ). Сравнение спектров поглощения этой пленки с НСПУНТ-3,4,5 четко показывает, что при размере пор НСПУНТ 4 нм и более профиль спектра в основном формируется спектром поглощения трубок, образующих структуру НСПУНТ. Четко выделяется резкий пик интенсивности на длине волны 1400 нм, который наблюдается в диапазоне 1300-1500 нм для моделей НСПУНТ-4,5. Резкие пики на длинах волн 460 и 540 нм проявляют себя и в интервале 500-650 нм НСПУНТ-3,4,5. Конечно, четкого совпадения по длинам волн нет для пиков интенсивности, но такого и не может наблюдаться, так как атомная структура НСПУНТ образована не параллельно расположенными ОУНТ, а их Х-контактами.
Таблица 2. Значения интегрального фототока исследуемых моделей НСПУНТ

\begin{tabular}{c|c|c}
\hline$N$ & $I_{\mathrm{ph}}, \mathrm{MA} \cdot \mathrm{cm}^{-2}(\mathrm{AM} 1.5)$ & $I_{\mathrm{ph}}, \mathrm{MA} \cdot \mathrm{cm}^{-2}(\mathrm{AM} 0)$ \\
\hline 0 & 2.41 & 3.25 \\
1 & 2.00 & 2.72 \\
2 & 1.71 & 2.31 \\
3 & 1.48 & 2.00 \\
4 & 1.12 & 1.55 \\
5 & 1.11 & 1.53
\end{tabular}

Для расчета спектра фототока $I_{\mathrm{ph}}$ использовалось известное распределение мощности солнечного излучения по длинам волн в диапазоне 100-2000 нм [27]. Максимум интенсивности солнечного излучения приходится на видимый диапазон. В направлении ИК длин волн интенсивность экспоненциально спадает для случая вне земной атмосферы - спектр АМ0 [27]. При наличии атмосферы Земли в спектре наблюдаются сильные провалы, вызванные наличием водных и других молекул в атмосфере - спектр АM1.5 [27]. Рассчитанные спектры максимального фототока с учетом спектров поглощения (рис. 4, $a$ ) и спектров интенсивности солнечного излучения представлены на рис. $4, b, c$. На рис. $4, b$ представлены спектры для случая атмосферы, на рис. 4, $c$ - вне атмосферы, например, на земной орбите. Профиль спектров фототока повторяет тенденции спектров поглощения. Максимальные значения фототока приходятся на середину видимого диапазона, поскольку именно в интервале 400-500 нм наблюдаются пики интенсивности поглощения, как видно из рис. 4, $a$. Значения интегрального фототока приведены в табл. 2. С увеличением размера поры НСПУНТ величина тока постепенно убывает, что объясняется уменьшением площади поглощения излучения. Необходимо отметить, что значения фототока для НСПУНТ-0 сравнимы со 
значениями для дисульфида молибдена $\mathrm{MoS}_{2}$, для которого вне атмосферы максимальный фототок составляет $3.9 \mathrm{MA} \cdot \mathrm{cm}^{-2}[28]$.

\section{5. Заключение}

Построены топологические модели суперьячеек наносетчатых пленок из углеродных нанотрубок с бесшовными крестообразными Х-соединениями. Установлено, что топология расположения негексагональных элементов в области контакта нанотрубок и их количество определяют энергетическую стабильность атомной структуры суперьячейки. Для наиболее выгодной с энергетической точки зрения структуры проведены исследования электропроводности и фотовольтаических свойств в зависимости от размеров поры пленки. Установлено, что размеры поры определяют тип проводимости. При наименьших размерах поры пленка проявляет почти металлические свойства с шириной щели $<0.1$ эВ. С увеличением размера поры щель в зонной структуре также увеличивается и пленка становится полупроводником. Управляя размером поры, таким образом, можно управлять электропроводностью пленки. Наличие щели в зонной структуре делает наносетчатые пленки очень перспективными для наноэлектроники и даже оптоэлектроники. Показано, что фототок для рассмотренных моделей пленки может достигать $2.4 \mathrm{MA} \cdot \mathrm{cm}^{-2}$ в условиях атмосферы и $3.25 \mathrm{MA} \cdot \mathrm{cm}^{-2}$ вне атмосферы. В сочетании с высокой эластичностью подобных пленок перечисленные электронные и оптоэлектронные свойства делают наносетчатые пленки хорошей основой для разработки элементной базы современных электронных наноустройств.

\section{Финансирование работы}

Работа выполнена при поддержке гранта Президента Российской Федерации для государственной поддержки научных исследований молодых российских ученых кандидатов наук МК-2373.2019.2.

\section{Конфликт интересов}

Авторы заявляют, что у них нет конфликта интересов.

\section{Список литературы}

[1] M. Engel, J.P. Small, M. Steiner, M. Freitag, A.A. Green, M.C. Hersam, P. Avouris. ACS Nano, 2 (12), 2445 (2008).

[2] X. Li, L. Zhang, X. Wang, I. Shimoyama, X. Sun, W.S. Seo, H. Dai. J. Am. Chem. Soc., 129, 4890 (2007).

[3] R. Du, S. Ssenyange, M. Aktary, M.T. McDermott. Small, 5 (10), 1162 (2009).

[4] Y.T. Liu, T.T. Yao, W.S. Zhang, G.P. Wu. Mater. Lett., 236, 244 (2019).

[5] Z. Lin, X. Gui, Q. Gan, W. Chen, X. Cheng, M. Liu, Y. Zhu, Y. Yang, A. Cao, Z. Tang. Sci. Rep., 5, 11336 (2015).
[6] J. Shi, X. Li, H. Cheng, Z. Liu, L. Zhao, T. Yang, Z. Dai, Z. Cheng, E. Shi, L. Yang, Z. Zhang, A. Cao, H. Zhu, Y. Fang. Adv. Funct. Mater., 26, 2078 (2016).

[7] H. Zhang, Y. Fu, C. Wang, P.C. Chen, Z. Liu, W. Wei, C. Wu, M.E. Thompson, C. Zhou. Nano Lett., 11, 4852 (2011).

[8] O. Kanoun, C. Müller, A. Benchirouf, A. Sanli, T.N. Dinh, A. Al-Hamry, L. Bu, C. Gerlach, A. Bouhamed. Sensors, 14, 10042 (2014).

[9] S. Aikawa, E. Einarsson, T. Thurakitseree, S. Chiashi, E. Nishikawa, S. Maruyama. Appl. Phys. Lett., 100, 063502 (2012).

[10] L. Cai, C. Wang. Nanoscale Res. Lett., 10, 320 (2015).

[11] H.J. Jeong, H.D. Jeong, H.Y. Kim, J.S. Kim, S.Y. Jeong, J.T. Han, D.S. Bang, G.W. Lee. Adv. Funct. Mater., 21, 1526 (2011).

[12] T. Kim, M. Cho, K.J. Yu. Materials, 11, 1163 (2018).

[13] Y. Li, X. Qiu, Y. Yin, F. Yang, Q. Fan. Phys. Lett. A, 374, 1773 (2010).

[14] L. Sun, X. He, J. Lu. npj Comput. Mater., 2, 16004 (2016).

[15] L. An, X. Yang, C. Chang. IJTAN, 1 (2), 30 (2013).

[16] M. Li, X. Liu, X. Zhao, F. Yang, X. Wang, Y. Li. Top. Curr. Chem. Z, 375, 29 (2017).

[17] G. Zhang, O.E. Glukhova. Comp. Mater. Sci., 184, 109943 (2020).

[18] J. Stuart, A.B. Tutein, J.A. Harrison. J. Chem. Phys., 112, 6472 (2000).

[19] M. Elstner, D. Porezag, G. Jungnickel, J. Elsner, M. Haugk, Th. Frauenheim, S. Suhai, G. Seifert. Phys. Rev. B, 58, 7260 (1998).

[20] O.E. Glukhova, M.M. Slepchenkov. J. Phys. Chem. C, 120 (31), 17753 (2016).

[21] M.M. Slepchenkov, D.S. Shmygin, G. Zhang, O.E. Glukhova. Nanoscale, 11 (35), 16414 (2019).

[22] S. Datta. Electronic Transport in Mesoscopic Systems (Cambridge University Press, Cambridge, 1995).

[23] O.E. Glukhova, D.S. Shmygin. Beilstein J. Nanotechnol., 9, 1254 (2018).

[24] H.A. Le, S.T. Ho, D.C. Nguyen, V.N. Do. J. Phys.: Condens. Matter, 26, 405304 (2014).

[25] O.E. Glukhova, I.S. Nefedov, A.S. Shalin, M.M. Slepchenkov. Beilstein J. Nanotechnol., 9, 1321 (2018).

[26] Y. Zhang, L. Meng, C. Yam, G. Chen. J. Phys. Chem. Lett., 5, 1272 (2014).

[27] С. Зи. Физика полупроводниковых приборов (М., Мир, 1984) т. 2, с. 391. [Пер. с англ.: S.M. Sze. Physics of semiconductor devices (N.Y., J. Wiley \& Sons, 1981) v. 2].

[28] M. Bernardi, M. Palummo, J. Grossman. Nano Lett., 13, 3664 (2013).

Редактор Л.В. Шаронова 


\section{Carbon nanotube nanomesh films with \\ $X$-shaped junctions for electronic and photovoltaic applications}

O.E. Glukhova, M.M. Slepchenkov, K.R. Asanov

Saratov State University,

410012 Saratov, Russia

Abstract The atomic structure, electronic and optoelectronic properties of nanosized films of carbon nanotubes with seamless cross-shaped X-junctions are studied. It is established that the topology of non-hexagonal elements arrangement in the contact region of nanotubes determines the energy stability of the atomic structure. It is revealed that the pore size of the film determines the type of conductivity. At the smallest pore sizes, the film is characterized by metallic type of conductivity; with an increase in pore size, the gap in the band structure also increases and the film becomes a semiconductor. Films with a minimum gap exhibit good photovoltaic characteristics. The photocurrent for the considered film models can reach $2.4 \mathrm{~mA} \cdot \mathrm{cm}^{-2}$ in the atmosphere and $3.25 \mathrm{~mA} \cdot \mathrm{cm}^{-2}$ outside the atmosphere. The presence of a gap in the band structure makes nanosized films promising for nanoelectronics and optoelectronics. 